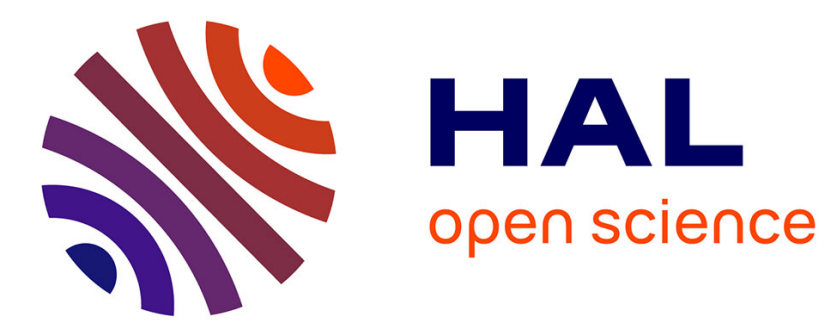

\title{
Étude théorique du couplage magnéto-élastique dans FeCl2 I. - Applications : propriétés a très basse température, $\mathrm{kT}$
}

\author{
J.A. Nasser
}

\section{> To cite this version:}

J.A. Nasser. Étude théorique du couplage magnéto-élastique dans FeCl2 I. - Applications : propriétés a très basse température, $\mathrm{kT}$

\section{HAL Id: jpa-00208104 https://hal.science/jpa-00208104}

Submitted on 1 Jan 1973

HAL is a multi-disciplinary open access archive for the deposit and dissemination of scientific research documents, whether they are published or not. The documents may come from teaching and research institutions in France or abroad, or from public or private research centers.
L'archive ouverte pluridisciplinaire HAL, est destinée au dépôt et à la diffusion de documents scientifiques de niveau recherche, publiés ou non, émanant des établissements d'enseignement et de recherche français ou étrangers, des laboratoires publics ou privés. 


\title{
Classification \\ Physics Abstracts \\ 17.60 \\ ÉTUDE THÉORIQUE DU COUPLAGE MAGNÉTO-ÉLASTIQUE DANS $\mathbf{F e C l}_{2}$ I. - APPLICATIONS : PROPRIÉTÉS A TRẼS BASSE TEMPÉRATURE, $k T \ll 12$ cm$^{-1}(*)$
}

\author{
J. A. NASSER \\ Service de Physique du Solide et de Résonance Magnétique \\ Centre d'Etudes Nucléaires de Saclay B P no 2, 91190 Gif-sur-Yvette, France
}

(Reçu le 23 mars 1973)

\begin{abstract}
Résumé. - Nous étudions le couplage magnéto-élastique dans $\mathrm{FeCl}_{2}$ à partir du potentiel thermodynamique du cristal, $G\left(T, \varepsilon_{\alpha}, m, \eta\right)$, fonction, à une température donnée, des formations du cristal $\varepsilon_{\alpha}$ et des paramètres magnétiques $m$ et $\eta$ qui permettent de décrire la transition antiferromagnétique. Après avoir montré l'origine des différents termes de $G$, nous utilisons $G$ pour étudier les effets magnéto-élastiques de $\mathrm{FeCl}_{2}$ à très basse température, $k T \ll 12 \mathrm{~cm}^{-1}$. Notre traitement permet de prévoir les variations des grandeurs magnétiques avec la température et avec les contraintes extérieures, ainsi qu'une magnétostriction lors de la transition du premier ordre antiferro-paramagnétique saturé.
\end{abstract}

Abstract. - We study the magneto-elastic coupling in $\mathrm{FeCl}_{2}$ by means of the Gibbs' free energy, of the crystal $G\left(T, \varepsilon_{\alpha}, m, \eta\right)$, a function, at a given temperature, of the crystal deformations $\varepsilon_{\alpha}$ and the magnetic variables $m$ and $\eta$ which enable us to describe the antiferromagnetic transition. After showing how the different terms of $G$ are arrived at, we use $G$ to study the magneto-elastic properties of $\mathrm{FeCl}_{2}$ at very low temperature, $k T \ll 12 \mathrm{~cm}^{-1}$. Our procedure enables us to determine the variations of the magnetic observables in terms of temperature and external constraints, as well as a magnetostriction at the first order transition from the antiferro- to the paramagnetic phase.

1. Introduction. - $\mathrm{FeCl}_{2}$ est un composé fortement ionique qui présente à basse température un ordre antiferromagnétique [1]. A ce jour ses propriétés magnétiques ont fait l'objet de nombreuses études expérimentales [2] à [13] qui s'interprètent correctement dans un modèle esquissé par Kanamori [14] et mis au point par Ôno et al. [2], [3]. Cependant, dans ces dernières années, des expériences montrent la possibilité d'un couplage magnéto-élastique [13], [15], [16], [17], [18].

Dans cet article nous présentons un modèle qui tient compte des résultats de Kanamori et d'Ôno et al. mais qui permet de rendre compte des différents aspects du couplage magnéto-élastique. Le but est d'obtenir le potentiel thermodynamique, $G$, du cristal, fonction à une température donnée des déformations du cristal et de paramètres magnétiques.

L'article comprend deux parties : dans la première nous donnons l'expression de $G$ en montrant l'origine de ses différents termes. Dans la seconde nous l'uti-

$\left(^{*}\right)$ Cet article fait partie de la thèse de Doctorat d'Etat présentée à l'Université de Paris VI par l'auteur. lisons par des techniques classiques de minimisation pour rendre compte des effets magnéto-élastiques prévisibles ou observés à très basse température. Dans un prochain article nous utiliserons ce modèle pour étudier les aspects du couplage magnéto-élastique au voisinage de la température d'ordre antiferromagnétique $T_{\text {Néel }}$.

Notre méthode de calcul des effets magnéto-élastiques à partir du potentiel thermodynamique est la même que celle qu'ont employée d'abord C. P. Bean et D. S. Rodbell [27], puis Tsallis [28]. Bean et Rodbell traitent le cas d'un cristal isotrope et d'un échange ferromagnétique entre spins $\frac{1}{2}$. Aussi n'utilisent-ils qu'un paramètre de déformation et un paramètre magnétique. Tsallis étend la méthode à un antiferromagnétique d'Ising $\left(S=\frac{1}{2}\right)$ à deux sous-réseaux. Aussi introduit-il deux paramètres magnétiques. Par contre sa description de la cohésion du cristal est incomplète puisqu'il ne tient pas compte de l'énergie cinétique des noyaux. Nous pensons que l'apport principal de notre travail par rapport aux deux précédents est de montrer l'origine des différents termes de $G$. Outre cet apport, le cas que nous traitons est compliqué par la basse symétrie du cristal, par la 
présence d'une anisotropie cristalline ni nulle [27], ni infinie [28], et enfin par la possibilité qu'à chaque ion magnétique d'occuper trois états et non pas deux.

2. Enthalpie libre variationnelle $G\left(T, \varepsilon_{\alpha}, m, \eta\right)$. Dans la théorie de Kanamori [14] et d'Ôno et al. [2], [3] : d'une part les états quantiques des électrons $3 \mathrm{~d}$ de fer sont indépendants de ceux des électrons de valence $4 \mathrm{~s}$ et $3 \mathrm{p}$ respectivement du fer et du chlore ; d'autre part les électrons $3 \mathrm{~d}$ restent fortement liés aux noyaux de fer tandis que les électrons de valence forment la liaison ionique $\mathrm{Fe}^{++} \mathrm{Cl}^{-}$.

A partir de cette description, on peut admettre en première approximation que la cohésion et la symétrie de $\mathrm{FeCl}_{2}$ sont celles d'un cristal formé d'ions $\mathrm{Fe}^{++}$ et $\mathrm{Cl}^{-}$, et que le couplage magnéto-élastique a pour origine les variations avec les déformations du cristal : du champ cristallin $V_{\mathrm{c}}$ des électrons $3 \mathrm{~d}$, ainsi que de l'échange qui couple entre eux les spins des électrons $3 \mathrm{~d}$ de deux ions fer différents. En fait, une analyse $\mathrm{du}$ hamiltonien $\mathcal{H} \mathrm{du}$ cristal montre que, outre l'échange, seule une partie du champ cristallin intervient dans le couplage magnéto-élastique. Cette analyse permet de décomposer $\mathscr{H}$ en deux :

- $\mathcal{H}_{\mathrm{R}}$ qui rend compte de la cohésion du cristal et auquel est rattachée la partie de $V_{\mathrm{c}}$ de symétrie cubique ;

- $\mathcal{H}_{\mathrm{me}}$ qui rend compte des propriétés magnétiques ainsi que de la possibilité d'un couplage magnétoélastique. $\varkappa_{\mathrm{me}}$ est tel que lorsqu'on néglige le couplage magnéto-élastique on retrouve le modèle de Kanamori et d'Ôno et al. caractérisé par un hamiltonien magnétique $\mathfrak{H}_{\mathrm{m}}$.

A $\mathcal{H}_{\mathbf{R}}$ on associe une énergie libre variationnelle $F_{\mathrm{R}}\left(T, \varepsilon_{\alpha}\right)$ qui à une température donnée a pour paramètres les déformations $\varepsilon_{\alpha}$. A $\mathcal{H}_{\mathrm{me}}$ on associe une enthalpie libre variationnelle $G_{\mathrm{me}}\left(T, \varepsilon_{\alpha}, m, \eta\right)$ qui à une température donnée a pour paramètres les déformations $\varepsilon_{\alpha}$ mais aussi les paramètres magnétiques $m$ et $\eta$ qui permettent de décrire la transition antiferromagnétique. L'enthalpie libre variationnelle $G \mathrm{du}$ cristal est alors la somme de $F_{\mathrm{R}}$ et de $G_{\text {me }}$.

Nous commençons par présenter les résultats de Kanamori et d'Ôno et al. qui nous servirons constamment de guide pour trouver $\mathcal{H}$ et $G$.

2.1 MODÈLE D'ÔNO et al. : HAMILTONIEN ET ENTHALPIE LIBRE MAGNÉTIQUe, $\mathfrak{H}_{\mathrm{m}}$ ET $G_{\mathrm{m}}$. - Le niveau fondamental de l'ion libre $\mathrm{Fe}^{++}$a pour termes spectraux $L=2$ et $S=2$. Dans le réseau cristallin de $\mathrm{FeCl}_{2}$, nous décrivons l'interaction des électrons $3 \mathrm{~d}$ d'un ion $\mathrm{Fe}^{++}$avec les autres ions et électrons du cristal par un champ cristallin de symétrie $\overline{3} \mathrm{~m}$ et un hamiltonien d'échange du type $\sum_{<i, j>}-2 J_{i j} \mathbf{S}_{i} \mathbf{S}_{j}$. On traite $V_{\mathrm{c}}$ et l'échange comme des perturbations dans le niveau fondamental de l'ion libre.

On peut décomposer $V_{\mathrm{c}}$ en la somme d'un opérateur de symétrie cubique $K$, et d'un opérateur de symétrie trigonale $T$. La diagonalisation de $K$ dans la représentation $\left\{\left|2, M_{L}\right\rangle\right\}$ donne un triplet fondamental $\Gamma_{5}$ séparé d'un doublet excité $\Gamma_{3}$ par $10^{4} \mathrm{~cm}^{-1}$ environ. Dans $\Gamma_{5}$ on peut définir un moment orbital fictif $\mathbf{l}=-\mathbf{L}$, avec $l=1$, et $\Gamma_{5}$ est sous-tendu par les kets $\mid l, m_{l}>$. Il est important de souligner que les $\mid l, m_{l}>$ sont des combinaisons linéaires des kets $\mid 2, M_{L}>$ indépendantes de la grandeur de $K$.

Dans la représentation $\left\{\left|l, m_{l}>\otimes\right| 2, M_{S}>\right\} T$ peut s'écrire $\Delta\left(l_{\mathrm{z}}^{2}-\frac{2}{3}\right)$ à des constantes additives près qui dépendent de la position des noyaux; $\Delta$ est un paramètre, et $l_{\mathrm{z}}$ est la projection de $\mathbf{l}$ sur l'axe $\mathbf{O z}$ choisi parallèle à l'axe ternaire. Il faut à présent diagonaliser le hamiltonien magnétique du cristal $\mathfrak{H}_{\mathrm{m}}$ dans la représentation $\left\{\left|1, m_{l}>\otimes\right| 2, M_{S}>\right\}$ $\mathcal{H}_{\mathrm{m}}$ est défini par

$\mathscr{H}_{\mathrm{m}}=\sum_{i} \mathcal{H}_{0}^{(i)}+\sum_{<i, j>}-2 J_{i j} \mathbf{S}_{i} \mathbf{S}_{j}-\sum \boldsymbol{\mu}^{(i)} \mathbf{H}$

avec

$$
\mathcal{H}_{0}^{(i)}=\lambda^{\prime}\left[\mathbf{l}_{i} \cdot \mathbf{S}_{i}-\frac{\Delta}{\lambda^{\prime}}\left(l_{i \mathrm{z}}^{2}-\frac{2}{3}\right)\right]
$$

où $\sum_{i}$ représente la somme sur les ions $\mathrm{Fe}^{++}$et $\sum_{<, j}$ celle sur les couples d'ions $\mathrm{Fe}^{++} ; \mathbf{S}_{i}$ est le spin total des électrons $3 \mathrm{~d}$ d'un ion $\mathrm{Fe}^{++} ; \lambda^{\prime}=-\lambda$, et $\lambda$ est le coefficient de couplage spin-orbite des électrons $3 \mathrm{~d}$ d'un ion $\mathrm{Fe}^{++} ; \mathbf{H}$ est le champ magnétique appliqué ; et $\boldsymbol{\mu}^{(i)}$ est le moment magnétique d'un ion $\mathrm{Fe}^{++}$, $\boldsymbol{\mu}^{(i)}=-\mu_{\mathrm{B}}\left(-\mathbf{l}^{(i)}+2 \mathbf{S}^{(i)}\right)$. Par ailleurs Ôno et al. ont établi que $\lambda^{\prime} \simeq \Delta \simeq 100 \mathrm{~cm}^{-1}$, alors que l'échange n'est que de l'ordre de $24 \mathrm{~K}$.

On diagonalise d'abord $\mathcal{H}_{0}^{(i)}$ qui commute avec $j_{\mathrm{z}}^{(i)}=l_{\mathrm{z}}^{(i)}+S_{\mathrm{z}}^{(i)}$. Leurs vecteurs propres communs ne dépendent que de $a=\Delta / \lambda^{\prime}$. De même, pour $\lambda^{\prime}$ fixé, la structure de niveau de $\mathfrak{H E}_{0}^{(i)}$, qui est rapportée dans les références [2], [3] ne dépend que de $a$. Pour chaque ion $\mathrm{Fe}^{++}$le niveau fondamental d'énergie $E$ est doublement dégénéré et a pour vecteurs propres $\pm 1>$ qui sont aussi vecteurs propres de $j_{z}$ avec les valeurs propres \pm 1 . Le premier niveau excité est un singulet situé à une distance $D$ de l'ordre de $10 \mathrm{~cm}^{-1}$ et dont le vecteur propre $\mid 0>$ est vecteur propre de $j_{\mathrm{z}}$ avec la valeur propre 0 . Les autres niveaux se trouvent à une distance comprise entre $2 \lambda^{\prime}$ et $5 \lambda^{\prime}$ soient 200 et $500 \mathrm{~cm}^{-1}$. On n'en tiendra pas compte dans les propriétés de $\mathrm{FeCl}_{2}$ à basse température, si $k T \ll 200 \mathrm{~cm}^{-1}$. Au premier ordre des perturbations, il faut diagonaliser l'échange et le terme Zeeman dans le sous-espace sous-tendu par $|0\rangle$ et $\mid \pm 1>$; c'est un cas de quasi-dégénérescence. Pour cela, on considère que chaque ion a un triplet fondamental d'énergie $E$ et de vecteurs propres $\mid \pm 1>$ et $\mid 0>$ sur lequel agit la perturbation :

$$
\sum_{i}-D\left(j_{z}^{2(i)}-1\right)-\sum_{<i, j>} 2 J_{i j} \mathbf{S}_{i} \mathbf{S}_{j}-\sum \boldsymbol{\mu}^{(i)} \mathbf{H} .
$$

Dans une approximation de champ moléculaire 
à deux sous-réseaux $(\gamma)$ et $(\delta)$, pour un champ $\mathbf{H}$ parallèle à l'axe $\mathrm{Oz}$, et pour les aimantations $x$ et $y$ des sous-réseaux parallèles à l'axe $\mathrm{Oz}$, l'hamiltonien d'un ion du sous-réseau $(\gamma)$ s'écrit :

$$
J^{(\gamma)}=-D\left(j_{z}^{(\gamma) 2}-1\right)-\left(J_{1} x+J_{2} y\right) j_{z}^{(\gamma)}-h j_{z}^{(\gamma)}
$$

avec dans le triplet

$$
\begin{aligned}
& S_{\mathrm{z}}=g_{\mathrm{z}}^{\prime} j_{\mathrm{z}} \text { et }-l_{\mathrm{z}}+2 S_{\mathrm{z}}=g_{\mathrm{z}} j_{\mathrm{z}} ; \\
& x=\left\langle j_{\mathrm{z}}^{(\gamma)}\right\rangle_{T} \quad \text { et } y=\left\langle j^{(\delta)}\right\rangle_{T} ;
\end{aligned}
$$

$J_{1}=g_{\mathrm{z}}^{\prime 2}\left(2 n_{1} J_{1}\right)$ où $\left(2 n_{1} J_{1}\right)$ est la constante d'échange ferromagnétique entre un ion $\mathrm{Fe}^{++}$et ses $n_{1}(=6)$ premiers voisins contenus dans le plan passant par l'ion et perpendiculaire à $\mathrm{Oz} ; J_{2}=g_{\mathrm{z}}^{\prime 2}\left(2 n_{2} J_{2}\right)$ où $\left(\begin{array}{lll}2 & n_{2} & J_{2}\end{array}\right)$ est la constante d'échange antiferromagnétique entre un ion $\mathrm{Fe}^{++}$et ses $n_{2}(=3+3)$ premiers voisins contenus dans deux plans perpendiculaires à $\mathrm{Oz}$ et situés de part et d'autre de l'ion ; $h=-g_{\mathrm{z}} \mu_{\mathrm{B}} H$ où $\mu_{\mathrm{B}}$ est le magnéton de Bohr et $H$ le module du champ magnétique.

Si on appelle $u$ et $v$ la probabilité d'occupation de l'état $|0\rangle$ respectivement pour un ion des sousréseaux $(\gamma)$ et $(\delta)$ on peut calculer une enthalpie libre variationnelle $G_{\mathrm{m}}$ en fonction de $T, u, v, x$ et $y$. Il est en fait préférable d'introduire l'aimantation $m$ et le paramètre d'ordre de la transition paramagnétique-antiferromagnétique $\eta$ définis par $2 m=x+y$ et $2 \eta=x-y$. Pour un cristal contenant $N$ ions $\mathrm{Fe}^{++}$on a les relations :

$$
\frac{1}{N} G_{\mathrm{m}}(T, m, \eta)=(U-T S)
$$

avec

$$
\begin{aligned}
U=\frac{D}{2}(u+v)-h m-\frac{1}{2}\left(A m^{2}+B \eta^{2}\right) \\
S=-\frac{k}{2}[u \log u+ \\
+\frac{1}{2}(1-u+m+\eta) \log \frac{1}{2}(1-u+m+\eta) \\
+\frac{1}{2}(1-u-m-\eta) \log \frac{1}{2}(1-u-m-\eta)+v \log v \\
+\frac{1}{2}(1-v+m-\eta) \log \frac{1}{2}(1-v+m-\eta) \\
\left.+\frac{1}{2}(1-v-m+\eta) \log \frac{1}{2}(1-v-m+\eta)\right]
\end{aligned}
$$

$U$ est la moyenne thermique de $\mathcal{H}^{(\gamma)}+\mathcal{H}^{(\delta)}$, et $S$ est l'entropie calculée à partir des probabilités d'occupation des états $|0\rangle$ et $\mid \pm 1>$ pour un ion de chaque sous-réseau. La minimisation de $G_{\mathrm{m}}(T, u, v, m, \eta)$ par rapport à $u, v, m$ et $\eta$ donne les équations sui- vantes dont les deux dernières sont les équations de champ moléculaire :

$$
\begin{gathered}
u=\left[1+2 \mathrm{e}^{\beta D} \operatorname{ch} \beta(A m+B \eta+h)\right]^{-1} \\
v=\left[1+2 \mathrm{e}^{\beta D} \operatorname{ch} \beta(A m-B \eta+h)\right]^{-1} \\
2 m=\frac{2 \operatorname{sh} \beta(A m+B \eta+h)}{\mathrm{e}^{-\beta D}+2 \operatorname{ch} \beta(A m+B \eta+h)}+ \\
\quad+\frac{2 \operatorname{sh} \beta(A m-B \eta+h)}{\mathrm{e}^{-\beta D}+2 \operatorname{ch} \beta(A m-B \eta+h)} \\
2 s=\frac{2 \operatorname{sh} \beta(A m+B \eta+h)}{\mathrm{e}^{-\beta D}+2 \operatorname{ch} \beta(A m+B \eta+h)}- \\
-\frac{2 \operatorname{sh} \beta(A m-B \eta+h)}{\mathrm{e}^{-\beta D}+2 \operatorname{ch} \beta(A m-B \eta+h)}
\end{gathered}
$$

avec $\beta=1 / k T, A=J_{1}+J_{2}$ et $B=J_{1}-J_{2}$. Outre $\lambda^{\prime}$ ce modèle ne contient que trois paramètres indépendants $2 n_{1} J_{1}, 2 n_{2} J_{2}$ et $a=\Delta / \lambda^{\prime}$ dont Ôno $e t$ al. [2], [3] ont trouvé les valeurs qui sont respectivement $6,6 \mathrm{~cm}^{-1},-0,73 \mathrm{~cm}^{-1}$, et 1,25 ; par ailleurs pour $\lambda^{\prime}$ ils donnent $95 \mathrm{~cm}^{-1}$.

Les résultats essentiels des équations de champ moléculaire sont :

- à la température de Néel, une transition du second ordre paramagnétique $\rightarrow$ antiferro (AF) avec, si $H=0, m=0$ et $\eta$ petit ;

- à $0 \mathrm{~K}$ dans la phase (AF), $m=0$ et $\eta=1$, si $H<H_{\mathrm{s}}(\simeq 10 \mathrm{kOe})$,

- à $0 \mathrm{~K}$ si $H>H_{\mathrm{s}}$, une transition du premier ordre antiferro $\rightarrow$ paramagnétique saturée avec $\eta=0$ et $m=1$ dans la phase (PS).

2.2 HAMILTONIEN DU CRISTAL : EXPRESSION ET DÉCOMPOSITION. - Le couplage magnéto-élastique est lié au déplacement des atomes. Pour en rendre compte, il faut donc introduire dans le modèle de Kanamori et d'Ôno et al. L'énergie cinétique des noyaux, $T_{\mathrm{N}}$. Or nous savons que la branche des phonons optiques des cristaux ioniques se trouve dans l'infrarouge. Donc, lors de la diagonalisation $\mathrm{du}$ hamiltonien $\mathcal{H} \mathrm{du}$ cristal, nous prendrons en compte $T_{\mathrm{N}}$ en même temps que $\mathcal{H}_{\mathrm{m}}$ (le spectre énergétique de $\mathcal{H}_{0}^{(i)}$ s'étale sur $\left.500 \mathrm{~cm}^{-1}\right)$.

On écrit $\mathcal{H}$ à partir des énergies cinétiques et potentielles électrostatiques des électrons $3 \mathrm{~d}$ et des électrons de valence ( $4 \mathrm{~s}$ et $3 \mathrm{p}$ ), ainsi que du couplage spin-orbite des électrons $3 \mathrm{~d}$. He peut être mis sous la forme :

$\mathscr{H}=\mathscr{C}_{\mathbf{N}}+\mathscr{H}_{\mathrm{ad}}\left(\mathbf{R}, \mathbf{r}_{\mathbf{v}}, \mathbf{r}_{\mathrm{d}}, \mathbf{p}_{\mathbf{v}}, \mathbf{p}_{\mathbf{d}}\right)=\mathscr{C}_{\mathbf{N}}+\mathscr{H}_{\mathbf{a d}}(\mathbf{R})$,

où $\mathscr{C}_{\mathrm{N}}$ est l'énergie cinétique des noyaux de fer et de chlore; $\mathbf{r}_{\mathbf{v}}$ et $\mathbf{p}_{\mathbf{v}}$ sont respectivement les positions et impulsions des électrons de valence, et $\mathbf{r}_{\mathrm{d}}$ et $\mathbf{p}_{\mathrm{d}}$ celles des électrons $3 \mathrm{~d}$ du fer ; $\mathbf{R}$ symbolise la position des noyaux.

Nous allons déduire $\mathscr{H}_{\mathrm{ad}}(\mathbf{R})$ à partir des résultats 
du modèle de Kanamori et d'Ôno et al. Puis nous diagonaliserons He dans l'approximation adiabatique [19], [20], [21].

Nous admettons qu'il existe une valeur $\mathbf{R}_{0}$ de $\mathbf{R}$ où la position des noyaux dans l'espace a la symétrie du réseau rhomboédrique $(\overline{3} \mathrm{~m})$; et où le niveau fondamental $U_{0}\left(\mathbf{R}_{0}\right)$ d'une partie $\mathscr{H}_{\mathrm{c}}\left(\mathbf{R}_{0}\right)$ de $\mathcal{H}_{\mathrm{ad}}\left(\mathbf{R}_{0}\right)$ a pour fonction propre $\varphi_{v}\left(\mathbf{R}_{0}, \mathbf{r}_{\mathrm{v}}\right) \varphi_{\mathrm{d}}\left(\mathbf{R}_{0}, \mathbf{r}_{\mathrm{d}}\right)$. Dans cette fonction :

- la factorisation signifie que les états quantiques des électrons $3 \mathrm{~d}$ sont indépendants de ceux des électrons de valence ;

$-\varphi_{\mathrm{v}}\left(\mathbf{R}_{0}, \mathbf{r}_{\mathrm{v}}\right)$ décrit la liaison ionique $\mathrm{Fe}^{++} \mathrm{Cl}^{-}$: elle est telle qu'il y ait une probabilité nulle pour qu'un électron de valence soit sur un noyau de fer et qu'elle s'identifie à une fonction atomique $3 p$ de l'ion $\mathrm{Cl}^{-}$au voisinage d'un noyau de chlore. Cette fonction est unique pour la valeur propre $U_{0}\left(\mathbf{R}_{0}\right)$. ket

$-\varphi_{\mathrm{d}}\left(\mathbf{R}_{0}, \mathbf{r}_{\mathrm{d}}\right)$ est la fonction correspondant au

$$
\prod_{i} \mid \mathbf{R}_{0}^{(i)}, l, S, m_{l}, M_{S}>.
$$

Dans ce ket $\mathbf{R}_{0}^{(i)}$ est la position de l'ion fer $(i)$ pour $\mathbf{R}_{0}$; et $l, S, m_{l}, M_{S}$ ont la même signification que dans le paragraphe 2.1. Donc $\varphi_{\mathrm{d}}\left(\mathbf{R}_{0}, \mathbf{r}_{\mathrm{v}}\right)$ symbolise un grand nombre de fonctions obtenues à partir de $l=1$, $S=2$ et $m_{l}=-1,0,1$ et $M_{S}=-2, \ldots, 2$. Aussi, la valeur propre $U_{0}\left(\mathbf{R}_{0}\right)$ est-elle fortement dégénérée à cause des états possibles des électrons $3 \mathrm{~d}$.

A partir des hypothèses que nous venons de présenter, on voit qu'on retrouve les résultats d'Ôno et al. $\mathrm{Si}$ on diagonalise dans le sous-espace propre correspondant à $U_{0}\left(\mathbf{R}_{0}\right)$ le hamiltonien $\mathcal{H}_{\mathrm{m}}\left(\mathbf{R}_{0}\right)$ obtenu à partir de $\mathcal{H}_{\mathrm{m}}$ (éq. (1)) en remplaçant $\Delta$, $\lambda, J_{i j}$ respectivement par $\Delta\left(\mathbf{R}_{0}\right), \lambda\left(\mathbf{R}_{0}\right)$ et $J_{i j}\left(\mathbf{R}_{0}\right)$ et en donnant à $\Delta\left(\mathbf{R}_{0}\right), \lambda\left(\mathbf{R}_{0}\right)$ et $J_{i j}\left(\mathbf{R}_{0}\right)$ les valeurs trouvées expérimentalement par Ôno et al. pour $\Delta, \lambda$ et $J_{i j}$. On pose alors par définition

et

$$
\mathscr{H}_{\mathrm{m}}\left(\mathbf{R}_{\mathbf{0}}\right)=\mathscr{H}_{\mathrm{m}}
$$

$$
\mathscr{H}_{\mathrm{ad}}\left(\mathbf{R}_{0}\right)=\mathscr{H}_{\mathrm{c}}\left(\mathbf{R}_{0}\right)+\mathscr{H}_{\mathrm{m}}\left(\mathbf{R}_{0}\right) .
$$

Avec cette définition, $\mathfrak{H}_{c}\left(\mathbf{R}_{0}\right)$ contient :

- l'énergie cinétique des électrons de valence et celle des électrons $3 \mathrm{~d}$;

- l'énergie potentielle électrostatique des noyaux ;

- l'énergie potentielle électrostatique des électrons de valence;

- l'énergie potentielle des électrons $3 \mathrm{~d}$ hormis celle qui se traduit par l'échange, et hormis la partie du champ cristallin contenue dans $\mathscr{H}_{\mathrm{m}}\left(\mathbf{R}_{0}\right)$, c'està-dire celle qui n'a pas la symétrie cubique.

Nous savons ( $\$ 2.1)$ qu'en ce qui concerne les électrons $3 \mathrm{~d}$, le premier état excité de $\mathfrak{H}_{\mathrm{c}}\left(\mathbf{R}_{0}\right)$ (éq. $\left(3^{\prime}\right)$ ) s'obtient en plaçant un ion fer dans un état de $\Gamma_{3}$ situé à près de $10^{4} \mathrm{~cm}^{-1}$ de $U_{0}\left(\mathbf{R}_{0}\right)$. En ce qui concerne les électrons de valence, nous admettons que le premier état excité de $\mathcal{H}_{\mathrm{c}}\left(\mathbf{R}_{0}\right)$ est à près de $10 \mathrm{eV}$ de $U_{0}\left(\mathbf{R}_{0}\right)$ (cristal ionique).

Pour $\mathbf{R}$ peu différent de $\mathbf{R}_{0}\left(\mathbf{R} \# \mathbf{R}_{0}\right)$ :

- l'énergie potentielle des électrons de valence varie de $\delta V_{\mathrm{v}}$;

- l'échange varie ;

- a priori, le coefficient $\lambda$ varie aussi ;

- le champ cristallin varie de $\delta V_{\mathrm{c}}=\delta K+\delta \Delta+v^{\prime}$ où $\delta K$ est la variation de la partie cubique, $\delta \Delta$ est celle du paramètre $\Delta$ et $v^{\prime}$ est un potentiel cristallin qui n'existe que, si pour $\mathbf{R}$, le groupe ponctuel de symétrie autour d'un ion fer est inférieur à $\overline{3} \mathrm{~m}$.

Par définition, nous posons pour $\mathbf{R} \# \mathbf{R}_{0}$ :

$$
\mathcal{H}_{\mathrm{c}}(\mathbf{R})=\mathcal{H}_{\mathrm{c}}\left(\mathbf{R}_{0}\right)+\delta K+\delta V_{\mathrm{v}}
$$

et

$$
\mathscr{H}_{\mathrm{me}}(\mathbf{R})=\mathscr{H}_{\mathrm{m}}\left(\mathbf{R}_{0}\right)+\delta \mathscr{H},
$$

d'où, d'après l'éq. (2),

$$
\mathscr{H}_{\mathrm{ad}}(\mathbf{R})=\mathscr{H}_{\mathrm{c}}(\mathbf{R})+\mathscr{H}_{\mathrm{me}}(\mathbf{R}) .
$$

Dans l'éq. (4'), $\delta$ Ye contient :

- la variation de l'échange,

- la variation de $\lambda$,

- la variation de $\delta V_{\mathrm{c}}$ hormis $\delta K$.

Pour $\mathbf{R}$ voisin ou égal à $\mathbf{R}_{0}$, le hamiltonien $d u$ cristal est donc (éq. (2) et (4')) :

$$
\mathscr{H}=\mathscr{C}_{\mathrm{N}}+\mathscr{H}_{\mathrm{c}}(\mathbf{R})+\mathcal{H}_{\mathrm{m}}\left(\mathbf{R}_{\mathbf{0}}\right)+\delta \mathcal{H} .
$$

Nous allons diagonaliser $H^{\prime}$ (éq. $\left(2^{\prime}\right)$ ) dans l'approximation adiabatique. On diagonalise d'abord $\mathscr{H}_{\mathrm{c}}(\mathbf{R})$ (éq. (4)), le terme le plus grand, en considérant $\mathbf{R}$ comme un paramètre. Soit $U_{0}(\mathbf{R})$ son niveau fondamental. Nous admettons que $U_{0}(\mathbf{R})$ passe par un minimum pour $\mathbf{R}_{0}, \mathbf{R}_{0}$ étant la valeur définie précédemment. Pour $\mathbf{R}$ voisin de $\mathbf{R}_{0}$, à la valeur propre $U_{0}(\mathbf{R})$ correspond toujours la fonction propre

$$
\varphi_{\mathrm{v}}\left(\mathbf{R}_{0}, \mathbf{r}_{\mathrm{v}}\right) \varphi_{\mathrm{d}}\left(\mathbf{R}_{0}, \mathbf{r}_{\mathrm{d}}\right) .
$$

En effet, d'une part $\delta V_{\mathrm{v}}$ reste petit devant la distance $(\simeq 10 \mathrm{eV})$ entre $U_{0}\left(\mathbf{R}_{0}\right)$ et le premier état excité des électrons de valence, d'autre part les kets $\left|l, S, m_{l}, M_{S}\right\rangle$ obtenus à partir des kets $\mid L, S, M_{L}, M_{S}>$ (voir $\S 2.1)$ ne dépendent pas du module de la partie cubique $K$ du champ cristallin. Les fonctions $\varphi_{\mathrm{v}}\left(\mathbf{R}_{0}, \mathbf{r}_{\mathrm{v}}\right) \varphi_{\mathrm{d}}\left(\mathbf{R}_{0}, \mathbf{r}_{\mathrm{d}}\right)$ sont donc de bonnes fonctions propres pour appliquer l'approximation adiabatique [19], [20], [21].

Après avoir diagonaliser $\mathcal{H}_{\mathrm{c}}(\mathbf{R})$ il faut tenir compte des autres termes de $\mathcal{H}$ (éq. (2)). Si l'on néglige $\delta \mathcal{H}$, pour finir de diagonaliser $\mathcal{H}$ il faut :

- d'une part trouver les fonctions propres, $\Phi(\mathbf{R})$ et les valeurs propres de $\mathscr{H}_{\mathrm{R}}$ défini par

$$
\mathscr{H}_{\mathbf{R}}=\mathscr{C}_{\mathbf{N}}+U_{0}(\mathbf{R}) ;
$$

- d'autre part diagonaliser $\mathscr{H}_{\mathrm{m}}\left(\mathbf{R}_{0}\right)$ dans le sousespace sous-tendu par les fonctions $\varphi_{\mathrm{v}}\left(\mathbf{R}_{0}, \mathbf{r}_{\mathrm{v}}\right) \varphi_{\mathrm{d}}\left(\mathbf{R}_{0}, \mathbf{r}_{\mathrm{d}}\right)$. 
Ces deux problèmes concernent des espaces d'états quantiques différents. Il y a découplage entre le mouvement des ions et les propriétés magnétiques du réseau. C'est le modèle de Kanamori et d'Ôno et Ito. $\mathrm{Si}$ on tient compte de $\delta$ Je il y a un couplage entre le mouvement des ions et les propriétés magnétiques du cristal. Et c'est l'objet de notre étude. Pour cela, dans cet article, nous employons une méthode d'approximation statistique variationnelle à partir d'un potentiel thermodynamique fonction d'un certain nombre de paramètres [22]. Cette méthode rend compte des effets statiques à l'équilibre thermodynamique, mais ne permet pas l'étude des effets dynamiques (propagation d'ultrasons, etc...). Nous allons à présent donner l'expression du potentiel thermodynamique variationnel.

2.3 ENThalpie libre du CRISTAL. - A $\mathcal{H}_{\mathbf{R}}$ on peut associer à la température $T$ une énergie libre variationnelle $F_{\mathrm{R}}\left(T, \varepsilon_{\alpha}\right)$ développable suivant les composantes $\varepsilon_{\alpha}(\alpha=1,2, \ldots, 6)$ du tenseur des déformations (déformations repérées par rapport à $\mathbf{R}_{0}$ ), chaque terme du développement étant invariant par toutes les opérations du groupe de symétrie du cristal ( $\overline{3} \mathrm{~m})$ [23]. D'où :

$$
\begin{aligned}
F_{\mathrm{R}}\left(T, \varepsilon_{\alpha}\right)=F_{\mathrm{R}}^{0}(T)+t_{1}(T)\left(\varepsilon_{1}\right. & \left.+\varepsilon_{2}\right)+t_{3}(T) \varepsilon_{3}+ \\
& +\frac{1}{2} \sum_{\alpha \beta} C_{\alpha \beta}(T) \varepsilon_{\alpha} \varepsilon_{\beta}+\cdots
\end{aligned}
$$

$C_{\alpha \beta}(T)$ sont les composantes d'un tenseur de rang 4, leurs relations de symétrie étant celles du tenseur d'élasticité rhomboédrique $(\overline{3} \mathrm{~m})$. $C_{\alpha \beta}$ et $\varepsilon_{\alpha}$ sont ici transcrits en notations matricielles, et sont définis sur trois axes orthogonaux $\mathrm{Ox}_{1}, \mathrm{x}_{2}, \mathrm{x}_{3}$ tels que $\mathbf{O} \mathbf{x}_{3}$ soit confondu avec l'axe ternaire. Pour les questions d'élasticité nous adoptons les notations et conventions de Nye [24]. La variation avec la température des invariants linéaires de ce développement traduit les propriétés de dilatation thermique; nous avons évidemment $t_{1}(0)=t_{3}(0)=0$. Les invariants linéaires proviennent des termes anharmoniques de $U_{0}(\mathbf{R})$ contenu dans l'éq. (5).

A $\mathcal{H}_{\mathrm{me}}$ on peut associer, à une température donnée $T$, une enthalpie libre $G_{\mathrm{me}}\left(T, \varepsilon_{\alpha}, m_{1}, m_{2}, \ldots\right)$ qu'on peut développer suivant les déformations $\varepsilon_{\alpha}$ et suivant des paramètres magnétiques $m_{1}, m_{2}$, etc...

Par la suite nous ne nous intéresserons qu'aux déformations qui respectent la symétrie du cristal ; les axes $\mathrm{Ox}_{1} \mathrm{x}_{2} \mathrm{x}_{3}$ sont alors les axes principaux de la quadrique des déformations et $\alpha=1,2$ ou 3 . Cette restriction permet cependant l'application du modèle pour les effets magnéto-élastiques suivants : comportement à basse température, $k T \ll 200 \mathrm{~cm}^{-1}$; magnétostriction à $T_{\mathrm{N}}(24 \mathrm{~K})$ qui se fait sans changement de symétrie [15]; comportement à basse température en présence d'un champ magnétique parallèle à l'axe ternaire; dans la phase PS, si le champ est perpendiculaire à l'axe, il doit être très petit pour qu'on puisse négliger les déformations qu'il produit et qui ne respectent pas la symétrie du cristal ; comportement à basse température en présence de contraintes extérieures qui respectent la symétrie du cristal, pression hydrostatique ou uniaxiale parallèle à l'axe ternaire.

Dans le cas où on ne s'intéresse qu'aux déformations qui respectent la symétrie du cristal, on admettra qu'on obtient $\mathscr{H}_{\text {me }}$ à partir de $\mathcal{H}_{\mathrm{m}}$ en y remplaçant $\Delta$ par $\Delta\left(\varepsilon_{\alpha}\right), \lambda^{\prime}$ restant constant, et les paramètres $2 n_{1} J_{1}$ et $2 n_{2} J_{2}$ par respectivement $2 n_{1} J_{1}\left(\varepsilon_{\alpha}\right)$ et $2 n_{2} J_{2}\left(\varepsilon_{\alpha}\right)$ avec par définition :

$$
Q\left(\varepsilon_{\alpha}\right)=Q(0)+\sum_{\alpha=1}^{3} \varepsilon_{\alpha} \frac{\partial Q}{\partial \varepsilon_{\alpha}}=Q(0)+\delta Q .
$$

$Q\left(\varepsilon_{\alpha}\right)$ représentant l'un des trois paramètres précédents. $Q(0)$ est la valeur de $Q\left(\varepsilon_{\alpha}\right)$ pour $\mathbf{R}=\mathbf{R}_{0}$ et les dérivées sont prises pour $\mathbf{R}=\mathbf{R}_{0}$ quel que soit $\varepsilon_{\alpha}$ supposé petit. Par raison de symétrie, on a toujours :

$$
\varepsilon_{1}=\varepsilon_{2} \quad \text { et } \quad\left(\frac{\partial}{\partial \varepsilon_{1}}-\frac{\partial}{\partial \varepsilon_{2}}\right)_{\mathbf{R}=\mathbf{R}_{0}} Q\left(\varepsilon_{\alpha}\right)=0 .
$$

Par ailleurs, comme $\lambda^{\prime}$ ne varie que de $5 \%$ lorsque l'ion $\mathrm{Fe}^{++}$passe de l'état d'ion libre $\left(\lambda^{\prime}=100 \mathrm{~cm}^{-1}\right)$ à l'état d'ion dans le réseau de $\mathrm{FeCl}_{2}\left(\lambda^{\prime}=95 \mathrm{~cm}^{-1}\right)$ il est légitime de penser que de petites déformations $\mathrm{du}$ réseau $\left(10^{-4}\right)$ n'affecteront pas sa valeur.

Dans ces conditions il faut remplacer $E$ par $E\left(\varepsilon_{\alpha}\right)$ et $G_{\mathrm{me}}$ s'obtient à partir de $G_{\mathrm{m}}$ en remplaçant $D, J_{1}$, $J_{2}$ et $h$ respectivement par $D\left(\varepsilon_{\alpha}\right), J_{1}\left(\varepsilon_{\alpha}\right), J_{2}\left(\varepsilon_{\alpha}\right)$ et $h\left(\varepsilon_{\alpha}\right)$ dont les définitions sont les mêmes que précédemment.

On admettra enfin que, dans nos conditions de travail, les paramètres $2 n_{1} J_{1}\left(\varepsilon_{\alpha}\right), 2 \quad n_{2} J_{2}\left(\varepsilon_{\alpha}\right)$ et $\Delta\left(\varepsilon_{\alpha}\right)$ sont bien représentés par leur développement au premier ordre, ce qui est compatible avec l'expérience [16], [17], [18]. Dans ces conditions, on peut vérifier que les coefficients $\partial^{2} G_{\mathrm{me}} / \partial \varepsilon_{\alpha} \partial \varepsilon_{\beta}$ sont nuls, ou négligeables devant les constantes élastiques mesurées [17]. Les $C_{\alpha \beta}$ sont donc les constantes élastiques par unité de volume du cristal, lesquelles ne dépendent pas de l'ordre magnétique.

On peut à présent donner l'expression de $G$, enthalpie libre variationnelle du cristal :

$G\left(T, \varepsilon_{\alpha}, m, \eta\right)=F_{\mathrm{R}}\left(T, \varepsilon_{\alpha}\right)+N E\left(\varepsilon_{\alpha}\right)+G_{\mathrm{me}}\left(T, \varepsilon_{\alpha}, m, \eta\right)$

avec

$$
\alpha=1,2,3 \text {. }
$$

$N$ étant le nombre d'ions $\mathrm{Fe}^{++}$par unité de volume.

A la température $T$, l'état d'équilibre le plus stable est tel que $G\left(T, \varepsilon_{\alpha}, m, \eta\right)$ soit minimal. La résolution des équations de minimisation permet d'exprimer les paramètres $\varepsilon_{\alpha}, \eta, m$ ainsi que toute grandeur physique en fonction uniquement de la température. Le nombre d'équations de minimisation est réduit à 4 par raison de symétrie entre $\varepsilon_{1}$ et $\varepsilon_{2}$. Enfin, les équations de minimisation par rapport à $\eta$ et $m$ donnent les équations de champ moléculaire écrites précédemment mais où les paramètres $D, A, B$ et $h$ sont remplacés par $D\left(\varepsilon_{\alpha}\right), A\left(\varepsilon_{\alpha}\right), B\left(\varepsilon_{\alpha}\right)$ et $h\left(\varepsilon_{\alpha}\right)$ dont 
les définitions sont évidentes (on a supposé faite la minimisation par rapport à $u$ et $v$ ).

Nous distinguerons dans l'utilisation de ce modèle deux domaines de température $T \ll T_{\text {Neel }}$ et $T \simeq T_{\text {Néel }}$ où $G_{\mathrm{me}}$ prend deux développements différents en fonction de $\eta$ et $m$.

2.4 Conclusion. - Nous avons pu donner l'origine des différents termes qui interviennent dans l'expression de l'enthalpie libre variationnelle de $\mathrm{FeCl}_{2}$. Nous pensons qu'une analyse semblable peut être entreprise pour d'autres composés magnétiques dont les électrons magnétiques ont des états quantiques indépendants de ceux des électrons de valence.

Notre modèle montre qu'on ne peut s'attendre à des effets magnéto-élastiques notables que si les dérivées par rapport aux déformations des paramètres d'échange et du paramètre d'anisotropie cristalline ne sont pas trop petites. Nous verrons par la suite que la cohésion du cristal intervient aussi par les constantes élastiques. En définitive nous devons introduire 11 paramètres: trois constantes élastiques $\left(C_{11}+C_{12}\right), C_{13}$ et $C_{33}$; deux dérivées pour chacun des deux paramètres de l'échange; deux dérivées pour le terme trigonal du champ cristallin; et enfin les deux paramètres $t_{1}(T)$ et $t_{3}(T)$ qui rendent compte de la dilatation thermique de cristal.

Enfin, il serait intéressant d'étendre ce modèle aux cax où les déformations ne respectent pas la symétrie initiale du cristal.

3. Applications. Propriétés de $\mathrm{FeCl}_{2}$ à très basse température $\left(k T \ll D\left(12 \mathrm{~cm}^{-1}\right)\right)$. - Nous avons vu précédemment (§ 2.1) qu'avant la diagonalisation des termes magnétiques - énergie d'échange et énergie Zeeman - le niveau fondamental d'un ion $\mathrm{Fe}^{++}$est un doublet situé à $D\left(\simeq 12 \mathrm{~cm}^{-1}\right)$ du premier niveau excité, un singulet. Pour cette raison, quand $T$ est différent de zéro et petit, il existe un domaine de température où seul l'état fondamental (en tenant compte des termes magnétiques) de chaque ion reste occupé. Aussi, dans cette zone de température, les solutions des équations de champ moléculaire restent-elles très voisines de celles à $0 \mathrm{~K}$; les termes correctifs se comportant comme $\mathrm{e}^{-2 \beta D}$. Si l'on prend dans cette zone de température, $k T \ll 2 D$, pour $\eta$ et $m$ les solutions à $0 \mathrm{~K}$, alors $G_{\mathrm{me}}\left(T, \varepsilon_{\alpha}, \eta, m\right)$ peut s'écrire :

- dans la phase AF : $\eta=1$ et $m=0$

$$
G_{\mathrm{me}}^{\mathrm{AF}}\left(T, \varepsilon_{\alpha}\right)=N\left[E\left(\varepsilon_{\alpha}\right)-\frac{J_{1}\left(\varepsilon_{\alpha}\right)-J_{2}\left(\varepsilon_{\alpha}\right)}{2}\right]
$$

— dans la phase PS : $\eta=0$ et $m=1$ pour $H>H_{\text {s }}$ $(\simeq 10 \mathrm{kOe})$

$$
G_{\mathrm{me}}^{\mathrm{PS}}\left(T, \varepsilon_{\alpha}\right)=N\left[E\left(\varepsilon_{\alpha}\right)-\frac{J_{1}\left(\varepsilon_{\alpha}\right)+J_{2}\left(\varepsilon_{\alpha}\right)}{2}+h\left(\varepsilon_{\alpha}\right)\right] .
$$

Ces expressions s'obtiennent donc après minimisation de $G\left(T, \varepsilon_{\alpha}, m, \eta\right)$ par rapport aux paramètres magnétiques $\eta$ et $m$. $G$ reste à une température donnée $T$ fonction des déformations $\varepsilon_{\alpha}$ et s'écrit :

- dans la phase AF :

$$
\begin{aligned}
& G^{\mathrm{AF}}\left(T, \varepsilon_{\alpha}\right)=F_{\mathrm{R}}^{0}(T)+t_{1}(T)\left(\varepsilon_{1}+\varepsilon_{2}\right)+t_{3}(T) \varepsilon_{3}+ \\
& \quad+\frac{1}{2} \sum_{\alpha=1}^{3} C_{\alpha \beta}(T) \varepsilon_{\alpha} \varepsilon_{\beta}+N\left[E\left(\varepsilon_{\alpha}\right)-\frac{J_{1}\left(\varepsilon_{\alpha}\right)-J_{2}\left(\varepsilon_{\alpha}\right)}{2}\right] \\
& - \text { dans la phase PS : } \\
& \quad G^{\mathrm{PS}}\left(T, \varepsilon_{\alpha}\right)= \\
& =F_{\mathrm{R}}^{\mathrm{O}}(T)+t_{1}(T)\left(\varepsilon_{1}+\varepsilon_{2}\right)+t_{3}(T) \varepsilon_{3}+\frac{1}{2} \sum_{\alpha=1}^{3} C_{\alpha \beta}(T) \varepsilon_{\alpha} \varepsilon_{\beta} \\
& \quad+N\left[E\left(\alpha_{\alpha}\right)-\frac{J_{1}\left(\varepsilon_{\alpha}\right)+J_{2}\left(\varepsilon_{\alpha}\right)}{2}+h\left(\varepsilon_{\alpha}\right)\right]
\end{aligned}
$$

soit encore

$G^{\mathrm{PS}}\left(T, \varepsilon_{\alpha}\right)=G^{\mathrm{AF}}\left(T, \varepsilon_{\alpha}\right)+N\left[-J_{2}\left(\varepsilon_{\alpha}\right)+h\left(\varepsilon_{\alpha}\right)\right]$ avec $t_{1}(0 \mathrm{~K})=t_{3}(0 \mathrm{~K})=0$.

Nous allons utiliser ces expressions de $G^{\mathrm{AF}}\left(T, \varepsilon_{\alpha}\right)$ et $G^{\mathrm{PS}}\left(T, \varepsilon_{\alpha}\right)$ pour déterminer les déformations du cristal à l'équilibre thermodynamique dans une phase donnée, à une température donnée et en présence ou non de contraintes mécaniques extérieures. Connaissant les déformations à l'équilibre thermodynamique, on peut prévoir les variations des grandeurs magnétiques en fonction de la température ou de la pression appliquée $P$. Ces variations sont des aspects $\mathrm{du}$ couplage magnéto-élastique.

3.1 LeS DÉFORMATIONS A L'ÉQUILIBRE THERMODYNAMIQUE. - 3.1.1 Equilibre à $0 \mathrm{~K}$ dans la phase AF. A $0 \mathrm{~K}$, les déformations qui correspondent à un équilibre stable sont celles qui minimisent la fonction des $\varepsilon_{\alpha}, G^{\mathrm{AF}}\left(0 \mathrm{~K}, \varepsilon_{\alpha}\right)$. Ce sont les solutions $\varepsilon_{1}^{0}, \varepsilon_{2}^{0}, \varepsilon_{3}^{0}$ des équations :

$$
\begin{aligned}
\left(C_{11}+C_{12}\right) & \varepsilon_{1}^{0}+C_{13} \varepsilon_{3}^{0}+ \\
+ & N\left[\frac{\partial E}{\partial \varepsilon_{1}}-\frac{1}{2}\left(\frac{\partial J_{1}}{\partial \varepsilon_{1}}-\frac{\partial J_{2}}{\partial \varepsilon_{1}}\right)\right]=0 \\
2 C_{13} \varepsilon_{1}^{0}+ & C_{33} \varepsilon_{3}^{0}+ \\
+ & N\left[\frac{\partial E}{\partial \varepsilon_{3}}-\frac{1}{2}\left(\frac{\partial J_{1}}{\partial \varepsilon_{3}}-\frac{\partial J_{2}}{\partial \varepsilon_{3}}\right)\right]=0
\end{aligned}
$$

Dans ces relations les constantes élastiques ont leur valeur à $0 \mathrm{~K}$; par ailleurs on rappelle que les dérivées partielles sont prises pour $\mathbf{R}=\mathbf{R}_{0}$.

Pour la suite on supposera que les constantes élastiques $C_{\alpha \beta}$ sont indépendantes de la température[17]. On prendra comme origine des déformations non plus la position des ions pour $\mathbf{R}=\mathbf{R}_{0}$, mais celle qui correspond à l'équilibre à $0 \mathrm{~K}$ dans la phase $\mathrm{AF}$. On admettra aussi que les valeurs $1,25,6,6,-0,73 \mathrm{~cm}^{-1}$, trouvées expérimentalement par Ôno et al. corres- 
pondent dans notre modèle aux valeurs à l'équilibre, à $0 \mathrm{~K}$, dans la phase $\mathrm{AF}$, des paramètres respectivement $a\left(\varepsilon_{\alpha}\right), 2 \quad n_{1} J_{1}\left(\varepsilon_{\alpha}\right), 2 \quad n_{2} J_{2}\left(\varepsilon_{\alpha}\right)$. On appellera $f_{0}$ la valeur à l'équilibre, à $0 \mathrm{~K}$, dans la phase $\mathrm{AF}$, de toute fonction $f\left(\varepsilon_{\alpha}\right)$ de ces paramètres. Ainsi :

$$
\begin{aligned}
& g_{z_{0}}=3,999 ; \quad g_{z_{0}}^{\prime 2}=2,777 ; \\
& J_{1_{0}}=g_{z_{0}}^{\prime 2}\left(2 n_{1} J_{1}\right)_{0}=18,33 \mathrm{~cm}^{-1} ; \\
& J_{2_{0}}=g_{z_{0}}^{\prime 2}\left(2 n_{2} J_{2}\right)_{0}=-2,03 \mathrm{~cm}^{-1} ;
\end{aligned}
$$

d'où

$$
\begin{aligned}
& A_{0}=J_{1_{0}}+J_{2_{0}}=16,0 \mathrm{~cm}^{-1} ; \\
& B_{0}=J_{1_{0}}-J_{20}=20,36 \mathrm{~cm}^{-1} ;
\end{aligned}
$$

et $h_{0}=-g_{\mathrm{z}_{0}} \mu_{\mathrm{B}} H=-0,187 \mathrm{~cm}^{-1}$ pour $1 \mathrm{kOe}$, etc...

3.1.2 Equilibre à $T \neq 0 \mathrm{~K}$ sans contrainte extérieure. - a) Phase AF. - A $T \neq 0 \mathrm{~K}$, les déformations qui correspondent à un équilibre stable sont celles qui minimisent la fonction des $\varepsilon_{\alpha}, G^{\mathrm{AF}}\left(T, \varepsilon_{\alpha}\right)$. Ces déformations $\varepsilon_{\alpha}^{\mathrm{eq}}(T)$ vérifient :

$$
\begin{array}{cl}
\left(C_{11}+C_{12}\right) \varepsilon_{1}^{\mathrm{eq}}(T)+C_{13} \varepsilon_{3}^{\mathrm{eq}}(T)+t_{1}(T) & =0 \\
2 C_{13} \varepsilon_{1}^{\mathrm{eq}}(T)+C_{33} \varepsilon_{3}^{\mathrm{eq}}(T)+t_{3}(T) & =0 \\
\varepsilon_{1}^{\mathrm{eq}}(T)=\varepsilon_{2}^{\mathrm{eq}}(T) &
\end{array}
$$

Nous avons pris pour origine des déformations $\varepsilon_{1}^{0}, \varepsilon_{2}^{0}, \varepsilon_{3}^{0}$ (éq. (6)). Les solutions sont

$$
\left.\begin{array}{l}
\varepsilon_{1}^{\mathrm{eq}}(T)=-\frac{C_{33}}{K} t_{1}(T)+\frac{C_{13}}{K} t_{3}(T) \\
\varepsilon_{3}^{\mathrm{eq}}(T)=-\frac{C_{11}+C_{12}}{K} t_{3}(T)+\frac{2 C_{13}}{K} t_{1}(T)
\end{array}\right\}
$$

avec

$$
K=C_{33}\left(C_{11}+C_{12}\right)-2 C_{13}^{2} .
$$

b) Phase PS. - A $T \mathrm{~K}$, les déformations qui correspondent à un équilibre stable sont celles qui minimisent la fonction des $\varepsilon_{\alpha}, G^{\mathrm{PS}}\left(T, \varepsilon_{\alpha}\right)$. Ces déformations sont les $\varepsilon_{\alpha}^{\text {eq }}$ précédents auxquels s'ajoutent des déformations supplémentaires $\Delta \varepsilon_{\alpha}(H)$ définies par:

$$
\left(C_{11}+C_{12}\right) \Delta \varepsilon_{1}(H)+C_{13} \Delta \varepsilon_{3}(H)+
$$

$$
+N\left[-\frac{\partial J_{2}}{\partial \varepsilon_{1}}+\frac{\partial h}{\partial \varepsilon_{1}}\right]=0
$$

$2 C_{13} \Delta \varepsilon_{1}(H)+C_{33} \Delta \varepsilon_{3}(H)+N\left[-\frac{\partial J_{2}}{\partial \varepsilon_{3}}+\frac{\partial h}{\partial \varepsilon_{3}}\right]=0$

$$
\Delta \varepsilon_{1}(H)=\Delta \varepsilon_{2}(H) .
$$

En fonction des paramètres $a\left(\varepsilon_{\alpha}\right), 2 n_{2} J_{2}\left(\varepsilon_{\alpha}\right), 2 n_{1} J_{1}\left(\varepsilon_{\alpha}\right)$, ces relations s'écrivent :

$$
\left.\begin{array}{l}
\Delta \varepsilon_{1}(H)=-\frac{C_{33}}{K} P_{1}+\frac{C_{13}}{K} P_{3} \\
\Delta \varepsilon_{3}(H)=-\frac{C_{11}+C_{12}}{K} P_{3}+\frac{2 C_{13}}{K} P_{1}
\end{array}\right\}
$$

avec

$$
\begin{aligned}
P_{1} & =N\left[-\frac{\partial J_{2}}{\partial \varepsilon_{1}}+\frac{\partial h}{\partial \varepsilon_{1}}\right] \\
& =N\left[(0,292-0,016 H) \frac{\partial a}{\partial \varepsilon_{1}}-2,78 \frac{\partial\left(2 n_{2} J_{2}\right)}{\partial \varepsilon_{1}}\right] \\
P_{3} & =N\left[-\frac{\partial J_{2}}{\partial \varepsilon_{3}}+\frac{\partial h}{\partial \varepsilon_{3}}\right] \\
& =N\left[(0,292-0,016 H) \frac{\partial a}{\partial \varepsilon_{3}}-2,78 \frac{\partial\left(2 n_{2} J_{2}\right)}{\partial \varepsilon_{3}}\right]
\end{aligned}
$$

dans ces expressions $H$ est en kOe. Dans l'approximation où les $C_{\alpha \beta}$ sont indépendants de $T$, les $\Delta \varepsilon_{\alpha}$ le sont également. Le modèle prévoit donc une magnétostriction au cours de la transition AF $\rightarrow$ PS. La dépendance de $P_{1}$ et $P_{3}$ à l'égard de $\partial a / \partial \varepsilon_{\alpha}$ a été évaluée numériquement en calculant leur variation lorsque $a$ passe de 1,25 à 1 . Nous pouvons donner un ordre de grandeur de $P_{3}$ à partir de nos résultats expérimentaux [17], [18] qui sont :

pour $\varepsilon_{3}=-10^{-4}$,

$\frac{\Delta\left(2 n_{2} J_{2}\right)}{2 n_{2} J_{2}}=2,2 \times 10^{-5}$ et $\frac{\Delta a}{a}=-4,5 \times 10^{-5}$.

D'où

$$
\frac{\partial a}{\partial \varepsilon_{3}}=0,56 \quad \text { et } \quad \frac{\partial\left(2 n_{2} J_{2}\right)}{\partial \varepsilon_{3}}=0,16 \mathrm{~cm}^{-1} .
$$

En prenant $N$ égal à $1,5 \times 10^{22}$ ions $\mathrm{Fe}^{++}$par $\mathrm{cm}^{-3}$, on trouve $P_{3} \#-3$ bar ; ce qui correspond avec notre définition de $P_{3}$ à une tension de traction de 3 bar suivant l'axe Oz. Par ailleurs on sait [17], [18] que le cristal se comprime de $10^{-4}$ suivant l'axe $\mathrm{Oz}$ pour une tension de compression de 1 bar appliquée suivant cet axe. On peut donc prévoir un allongement relatif de quelque $10^{-4}$ suivant l'axe $\mathrm{c}$ lorsque le cristal passe de la phase AF à la phase PS.

3.1.3 Equilibre à $T \neq 0 \mathrm{~K}$ d'un échantillon soumis à une contrainte hydrostatique ou uniaxiale suivant l'axe ternaire. - A $T \neq 0 \mathrm{~K}$, les déformations qui correspondent à un équilibre stable en présence d'une pression hydrostatique $P$ sont celles qui vérifient

$$
\frac{\partial G\left(T, \varepsilon_{\alpha}\right)}{\partial \varepsilon_{\alpha}}=-P
$$

avec $\alpha=1,2,3$ et $G=G^{\mathrm{AF}}$ ou $G^{\mathrm{PS}}$.

Les solutions de ces équations sont, suivant la phase d'équilibre, les déformations $\varepsilon_{\alpha}^{\text {eq }}(T)$ ou $\varepsilon_{\alpha}^{\text {eq }}(T)+\Delta \varepsilon_{\alpha}(H)$ précédemment calculées, auxquelles s'ajoutent des déformations supplémentaires $\delta \varepsilon_{\alpha}(P)$. Puisque nous avons supposé les constantes élastiques indépendantes de l'ordre magnétique, les $\delta \varepsilon_{\alpha}(P)$ sont les mêmes dans les deux phases et sont définies par les relations :

avec

$$
\left.\begin{array}{c}
\left(C_{11}+C_{12}\right) \delta \varepsilon_{1}(P)+C_{13} \delta \varepsilon_{3}(P)=-P \\
2 C_{13} \delta \varepsilon_{1}(P)+C_{33} \delta \varepsilon_{3}(P)=-P \\
\delta \varepsilon_{1}(P)=\delta \varepsilon_{3}(P)
\end{array}\right\}
$$


On retrouve donc les relations classiques de l'élasticité valables à toute température. Dans le cas d'une pression uniaxiale, il suffit d'annuler le second membre de la deuxième relation.

On en conclut qu'à l'équilibre thermodynamique, à la température $T(k T \ll 2 D)$ en présence d'une contrainte extérieure $P$ et d'un champ magnétique $H$ les déformations sont :

- dans la phase AF

$$
\left.\varepsilon_{\alpha}^{\mathrm{eq}}(T)+\delta \varepsilon_{\alpha}(P) \quad \text { (éq. (7) }+(9)\right)
$$

- dans la phase PS

$$
\left.\varepsilon_{\alpha}^{\mathrm{eq}}(T)+\Delta \varepsilon_{\alpha}(H)+\delta \varepsilon_{\alpha}(P) \quad \text { (éq. (7) }+(8)+(9)\right)
$$

3.2 VARIATION DES OBSERVABLES MAGNÉTIQUES AVEC LA PRESSION $P$ OU AVEC LA TEMPÉRATURE POUR $k T \ll 2 D$. - En supposant que les ions $\mathrm{Fe}^{++}$des deux sous-réseaux occupent uniquement leur état fondamental (ce qui est valable si $k T \ll 2 D$ ) on peut exprimer les grandeurs magnétiques $M$ en fonction des paramètres $a\left(\varepsilon_{\alpha}\right), 2 \quad n_{2} J_{2}\left(\varepsilon_{\alpha}\right), 2 \quad n_{1} J_{1}\left(\varepsilon_{\alpha}\right)$. En remplaçant $\varepsilon_{\alpha}$ par les valeurs à l'équilibre thermodynamique, valeurs établies précédemment, on voit que $M$ n'est fonction que de $T, P$ et $H$. On peut écrire :

$$
\begin{aligned}
& M(T, P, H)= \\
& \quad=M\left[a_{0},\left(2 n_{1} J_{1}\right)_{0},\left(2 n_{2} J_{2}\right)_{0}\right]+\delta M(T, P, H),
\end{aligned}
$$

où $\delta M(T, P, H)$ dépend de $T, P, H$ par l'intermédiaire des coefficients de couplage magnéto-élastique $\partial Q\left(\varepsilon_{\alpha}\right) / \partial \varepsilon_{\alpha}$ et des constantes élastiques. On peut donc trouver des relations entre les coefficients de couplage magnéto-élastique, et entre ceux-ci et les constantes élastiques, en mesurant $\delta M$ dans deux types de conditions expérimentales :

- soit fixer la température et soumettre le cristal à une pression hydrostatique ou uniaxiale (ce type d'expérience peut être fait aussi à température plus haute mais l'expression de $M$ risque d'être plus compliquée que lorsqu'on suppose $k T \ll 2 D$ );

- soit laisser le cristal sous pression atmosphérique et faire varier sa température par exemple entre 1 et $6 \mathrm{~K}$.

Dans les expériences sous contraintes mécaniques $P$, à température constante, $\delta M$ varie linéairement avec $P$ (dans le cas où $P$ n'est pas trop grand). Trois mesures ont déjà été faites [16], [17], [18], et nous ont donné les valeurs numériques utilisées pour évaluer l'ordre de grandeur de $P_{3}$.

Lorsqu'on mesure dans la phase PS la susceptibilité magnétique, $\chi_{\perp}$, dans une direction perpendiculaire à l'axe d'anisotropie $\mathrm{Oz}$, il est important que le champ magnétique appliqué perpendiculairement à l'axe ne soit pas trop grand. En effet, un champ perpendiculaire à l'axe $\mathrm{Oz}$ crée des déformations dont la quadrique n'admet pas les axes $O x_{1} \quad x_{2} \quad x_{3}$ comme axes principaux. On peut en trouver une preuve dans les expériences de $\mathbf{M}$. K. Wilkinson et al. [1] qui montrent qu'un champ magnétique suffisamment fort provoque la destruction de $\mathrm{FeCl}_{2}$; ce qui est certainement dû à l'existence de contraintes de cisaillement auxquelles ce cristal lamellaire ne peut résister.

Dans les expériences à la pression atmosphérique et à température variable, $\delta M$ varie à cause de la dilatation thermique du cristal $\left[t_{1}(T)\right.$ et $\left.t_{3}(T)\right]$. Nous cherchons actuellement à la mesurer aux rayons $X$. Elle doit exister même à basse température puisque D. P. Johnson et J. G. Dash [25] concluent à partir d'expériences d'effet Mössbauer sur $\mathrm{FeCl}_{2}$ que les termes anharmoniques du potentiel internucléaire ne sont pas négligeables même à basse température.

Dans ces expériences où l'on mesure la variation thermique de $\delta M$ il peut être difficile d'évaluer pour certaines grandeurs la contribution de $\mathrm{Fe}^{3+}$ présent sous forme d'impureté comme l'ont signalé Masseyuki, Date et al. [26]. Nous ne connaissons pas la concentration de $\mathrm{Fe}^{3+}$ dans nos échantillons de $\mathrm{FeCl}_{2}$ mais Date et al. pensent que même dans les meilleurs cristaux de $\mathrm{FeCl}_{2}$ il est inévitable d'en avoir $1 \%$. Nous utiliserons leurs résultats de RPE sur $\mathrm{Fe}^{3+}$ dans $\mathrm{FeCl}_{2}$ pour discuter les mesures de chaleur spécifique et de susceptibilité magnétiques faites à très basse température par Carrara [13]. Mais au préalable nous allons établir l'expression dans notre modèle de la valeur $H_{\mathrm{s}}$ du champ magnétique qui induit la transition $\mathrm{AF} \rightarrow$ PS.

3.2.1 Champ seuil $\boldsymbol{H}_{\mathrm{s}}$ de la transition AF $\rightarrow$ PS. Appelons $\varepsilon_{\alpha}^{\prime}$ les déformations à l'équilibre dans la phase $\mathrm{AF}$ à la température $T$ d'un cristal soumis ou non à une contrainte mécanique extérieure. Nous avons vu que les déformations à l'équilibre dans la phase PS sont alors $\varepsilon_{\alpha}^{\prime}+\Delta \varepsilon_{\alpha}$. Avec ces notations, la valeur $H_{\mathrm{s}}$ du champ magnétique appliqué parallèlement à $\mathrm{Oz}$ pour laquelle a lieu la transition $\mathrm{AF} \rightarrow$ PS est définie par

$$
G^{\mathrm{AF}}\left(T, \varepsilon_{\alpha}^{\prime}\right)=G^{\mathrm{PS}}\left(T, \varepsilon_{\alpha}^{\prime}+\Delta \varepsilon_{\alpha}\right) .
$$

En utilisant la relation qui existe entre $G^{\mathrm{PS}}$ et $G^{\mathrm{AF}}$, et en utilisant le fait que les $\varepsilon_{\alpha}^{\prime}$ minimisent $G^{\mathrm{AF}}\left(T, \varepsilon_{\alpha}\right)$ on montre que $H_{\mathrm{s}}$ doit vérifier la relation :

$$
\begin{aligned}
{\left[-J_{2}\left(\varepsilon_{\alpha}^{\prime}\right)+h\left(\varepsilon_{\alpha}^{\prime}\right)\right]+\Delta \varepsilon_{1} } & \left(-\frac{\partial J_{2}}{\partial \varepsilon_{1}}+\frac{\partial h}{\partial \varepsilon_{1}}\right)+ \\
& +\frac{1}{2} \Delta \varepsilon_{3}\left(-\frac{\partial J_{2}}{\partial \varepsilon_{3}}+\frac{\partial h}{\partial \varepsilon}\right)=0
\end{aligned}
$$

dont les deux derniers termes sont des polynômes du second degré en $H$ à coefficients constants, et où $J_{2}\left(\varepsilon_{\alpha}^{\prime}\right)$ et $h\left(\varepsilon_{\alpha}^{\prime}\right)$ doivent être développés au premier ordre. Dans cette formule $\varepsilon_{\alpha}^{\prime}=\varepsilon_{\alpha}^{\text {eq }}(T)+\delta \varepsilon_{\alpha}(P)$ dans le cas général.

Lorsqu'on applique une pression suffisamment 
grande ( $\simeq 100$ bar) $\Delta \varepsilon_{1}$ et $\Delta \varepsilon_{3}$ peuvent être négligés devant $\delta \varepsilon_{\alpha}(P)$ et la formule devient :

$$
-J_{2}\left(\varepsilon_{\alpha}^{\mathrm{eq}}(T)+\delta \varepsilon_{\alpha}(P)\right)=-h\left(\varepsilon_{\alpha}(T)+\delta \varepsilon_{\alpha}(P)\right) .
$$

C'est la relation que nous avons employée pour interpréter les expériences rapportées en [17]. Il faut la développer ou la différencier logarithmiquement en considérant que $\delta \varepsilon_{\alpha}(P)$ est un accroissement.

Lorsque à la pression atmosphérique, on fait varier la température, nous pensons qu'on peut encore négliger les termes en $\Delta \varepsilon_{1}$ et $\Delta \varepsilon_{3}$.

3.2.2 Variation thermique de la chaleur spécifique $C(T)$ et de la susceptibilité suivant $\mathrm{Oz}, \chi_{\|}(T)$, dans la phase AF. - On peut prendre pour la susceptiblité suivant $\mathrm{Oz}$ l'expression [13] :

$$
\chi_{\|}\left(\varepsilon_{\alpha}\right)=N \frac{\mu_{\mathrm{B}}^{2}}{\lambda^{\prime}} 4,05\left[1+0,037 a\left(\varepsilon_{\alpha}\right)\right] .
$$

Cette expression est obtenue par un calcul de perturbation au second ordre en tenant compte des niveaux supérieurs de $\mathcal{H}_{0}^{(i)}$. Elle n'est valable que pour $a\left(\varepsilon_{\alpha}\right) \# a_{0} \ll 1$, ce qui n'est pas le cas dans $\mathrm{FeCl}_{2}$ où $a_{0}=1,25$. Nous pouvons cependant l'employer pour la discussion que nous allons faire. On en déduit :

$$
\begin{aligned}
& \left(\chi_{\|}\right)_{0}=N \frac{\mu_{\mathrm{B}}^{2}}{\lambda^{\prime}} 4,05\left(1+0,037 a_{0}\right) \\
& \delta \chi_{\|}(T)=N \frac{\mu_{\mathrm{B}}^{2}}{\lambda^{\prime}} 4,05 \times 0,037 \sum_{\alpha=1}^{3} \varepsilon_{\alpha}^{\mathrm{eq}}(T) \frac{\partial a}{\partial \varepsilon_{\alpha}}
\end{aligned}
$$

$\delta \chi_{\|}(T)$ varie donc avec $T$ comme $t_{1}(T)$ et $t_{3}(T)$ auxquels il est proportionnel.

La chaleur spécifique magnétique $C(T)$ se déduit de l'énergie magnétique $N U(T)$ par la relation $C(T)=N \mathrm{~d} U / \mathrm{d} T$. Or dans notre approximation $k T \ll 2 D, U$ est égal à :

$$
U\left(\varepsilon_{\alpha}\right)=E\left(\varepsilon_{\alpha}\right)-\frac{1}{2}\left[J_{1}\left(\varepsilon_{\alpha}\right)-J_{2}\left(\varepsilon_{\alpha}\right)\right],
$$

d'où l'on tire

$$
\begin{gathered}
U_{0}=E_{0}-\frac{1}{2}\left(J_{1_{0}}-J_{2_{0}}\right) \\
\delta U(T)=\sum_{\alpha=1}^{3} \varepsilon_{\alpha}^{\mathrm{eq}}(T) \frac{\partial E}{\partial \varepsilon_{\alpha}}-\frac{1}{2} \sum_{\alpha=1}^{3} \varepsilon_{\alpha}^{\mathrm{eq}}(T) \frac{\partial}{\partial \varepsilon_{\alpha}}\left(J_{1}-J_{2}\right) .
\end{gathered}
$$

On voit que $\delta U(T)$ varie avec $T$ comme $t_{1}(T)$ et $t_{3}(T)$. D'où $C(T)$ varie avec $T$ comme la dérivée de ces paramètres.

Par ailleurs nous avons les résultats expérimentaux suivants dans la phase AF [13] :

$\chi_{\|}(T)=\left(2,073 \times 10^{-2}+0,58 \times 10^{-4} T^{2}\right) \mathrm{cgs} / \mathrm{mole}$

$$
\text { pour } 1 \mathrm{~K} \leqslant T \leqslant 4,5 \mathrm{~K}
$$

$C(T)=1,10 \times 10^{-2} T$ joules $/$ mole entre 1 et $2 \mathrm{~K}$.

Pour interpréter ces résultats, on peut songer à prendre $t_{1}(T)$ ou/et $t_{3}(T)$ comme une forme quadratique de $T ; \delta \chi_{\|}(T)$ varierait comme $T^{2}$ et $C(T)$ comme $T$. On peut alors évaluer un ordre de grandeur de la dilatation thermique du cristal en supposant

$$
\varepsilon_{1}^{\mathrm{eq}}(T) \ll \varepsilon_{3}^{\mathrm{eq}}(T) .
$$

En remplaçant dans $\delta \chi_{\|}(T)$ on obtient :

$$
\delta \chi_{\|}(T)=N \frac{\mu_{\mathrm{B}}^{2}}{\lambda^{\prime}} 4,05\left(2 \times 10^{-2}\right) \varepsilon_{3}^{\mathrm{eq}}(T) .
$$

A partir du rapport $\delta \chi_{\|}(T) /\left(\chi_{\|}\right)_{0}$, en prenant pour $\left(\chi_{\|}\right)_{0}$ la valeur de $2,073 \times 10^{-2} \mathrm{cgs} / \mathrm{mole}$, on trouve :

$$
\varepsilon_{3}^{\mathrm{eq}}(T)=0,15 T^{2} \text {. }
$$

Ce résultat est impossible : dès $1 \mathrm{~K}$ le cristal se dilaterait de plus de 0,1 .

Ce résultat confirme le rôle des impuretés $\mathrm{Fe}^{3+}$ dont la contribution ne peut pas être négligée [26]. Nous pouvons évaluer l'ordre de grandeur de la chaleur spécifique et de la susceptibilité magnétique de $1 \%$ d'impuretés paramagnétiques de spin $S=\frac{5}{2}$ placées dans un champ magnétique effectif de 14,6 kOe parallèle à $\mathrm{Oz}$. Ce champ mesuré par Date et al. [26] est dû à l'ordre magnétique du réseau $\mathrm{Fe}^{2+}$. Nous avons calculé les valeurs suivantes à $2 \mathrm{~K}$ :

$$
\chi_{\|}\left(\mathrm{Fe}^{3+}\right)=5,8 \times 10^{-4} \mathrm{cgs}
$$

et

$$
C\left(\mathrm{Fe}^{3+}\right)=6,6 \times 10^{-3} \text { joule } / \mathrm{dg} .
$$

Ces valeurs sont proches des résultats expérimentaux précédents (éq. (10)), respectivement $2,3 \times 10^{-4} \mathrm{cgs}$ et $22 \times 10^{-3}$ joule/dg. Pour utiliser notre modèle, il est donc nécessaire d'éliminer au préalable la contribution de $\mathrm{Fe}^{3+}$ aux résultats expérimentaux des éq. (10).

3.3 Conclusion. - Notre modèle laisse prévoir une magnétostriction, au cours de la transition métamagnétique, observable aux rayons $\mathrm{X}$ à $4,2 \mathrm{~K}$.

Par ailleurs nous avons montré que l'on peut mesurer les coefficients de couplage magnéto-élastique à partir de deux types d'expérience. Dans les mesures à pression fixe et température variable, il est en fait facile d'éliminer la contribution des impuretés $\mathrm{Fe}^{3+}$ à partir des résultats de Date [26], et de la mesure de la concentration des impuretés. Dans celles à température fixe et pression variable, si l'on admet que le champ effectif agissant sur $\mathrm{Fe}^{3+}$ a des variations avec les déformations du même ordre de grandeur que celles des inter-actions magnétiques, alors la contribution des impuretés aux résultats expérimentaux reste de l'ordre de leur concentration; soit $1 \%$, ce qui est négligeable. Il serait d'ailleurs intéressant de refaire les expériences de Date et al. dans $\mathrm{FeCl}_{2}$ sous pression hydrostatique ou uniaxiale.

Remerciements. - Je remercie $M^{\text {me }} F$. HartmannBoutron et M. J. Hammann d'avoir lu l'article en manuscrit. Que MM. G. Sarma et N. Boccara qui ont toujours été pour moi accueillants et de bon conseil trouvent ici l'expression de mon amicale reconnaissance. 


\section{Bibliographie}

[1] Wilkinson, M. K., Cable, J. W., Wollan, E. O., Koehler, W. C., Phys. Rev. 113 (1959) 497.

[2] ÔNo, K., Iтo, A., Fuıita, T., J. Phys. Soc. Jap. 19 (1964) 2119.

[3] Fujita, T., Ito, A., ÔNo, K., J. Phys. Soc. Jap. 27 (1969) 1143.

[4] Jacobs, I. S., Lawrence, P. E., Phys. Rev. 164 (1967) 866.

[5] Jacobs, I. S., Roberts, S., Lawrence, P. E., Phys. Rev. 36 (1965).

[6] TrapP, C., Thesis, University of Chicago (1963), inédite.

[7] Trapeznikow, O., Schubnikow, L., Physik Z. Sowjet 7 (1935) 66.

[8] Stark, C., Bitter, F., Kaufmann, A. R., Phys. Rev. 58 (1940) 977

[9] Brandt, C. L. W., Thesis, University of Chicago (1960), inédite.

[10] Bizette, H., Terrïer, C., Tsai, B., C. R. Hebd. Séan. Acad. Sci. 261 (1965) 653.

[11] Hautecler, S., Konstantinovic, J., Cribier, D., Jacrot, B., C. R. Hebd. Séan. Acad. Sci. 254 (1962) 1026.

[12] Kostryukova, M. O., Sov. Phys. JETP 19 (1964) 1084.

[13] Carrara, P., Thèse, Université de Paris (1968), inédite.
[14] Kanamori, J., Progr. Theoret. Phys. 20 (1958) 890.

[15] Kleinberger, P., Nasser, J. A., à publier.

[16] Narath, A., Schirber, J. E., J. Appl. Phys. 37 (1966) 1124.

[17] Nasser, J. A., soumis à J. Phys. and Chem. Solids.

[18] Hammann, J., Nasser, J. A., Phys. Stat. Sol. (b) 56 (1973) 95.

[19] Born, M., Huang, K., Dynamical Theory of Crystal Lattices (Clarendon Press, Oxford).

[20] Messiah, A., Mécanique Quantique, Tome II (Dunod, Paris) 1960.

[21] Landau, L., Lifchitz, E., Physique Théorique, Tome III, Mécanique Quantique, $2^{\mathrm{e}}$ éd. (édit. MIR, Moscou) 1967.

[22] Boccara, N., Les Principes de la Thermodynamique Classique (PUF, Collection Sup.).

[23] Boccara, N., Ann. Phys. 47 (1968) 40-64.

[24] Nye, J. F., Propriétés Physiques des Cristaux (Dunod, Paris) 1961.

[25] Johnson, D. J. et Dash, J. G., Phys. Rev. 172 (1968) 983.

[26] Date, M., Motokawa, M., Phys. Rev. Lett. 15 (1965) 854.

[26 bis] Date, M., Motokawa, M., J. Phys. Soc. Jap. 23 (1967) 1216.

[27] Bean, C. P., Rodbell, D. S., Phys. Rev. 126 (1962) 104.

[28] Tsallis, C., J. Physique 32 (1971) 903. 